\title{
The Treatment of Female Pattern Hair Loss with Long Acting Platelet Rich Plasma
}

\author{
Benjamin C Paul, MD* \\ Facial Plastic and Hair Restoration Surgeon, USA \\ Received: 觜: October 26, 2018; Published: 笽: November 14, 2018 \\ *Corresponding author: Benjamin C. Paul, MD, Facial Plastic and Hair Restoration Surgeon, USA
}

\section{Opinion}

Female Pattern Hair Loss (FPHL), also referred to as Androgenic Alopecia (AGA), is the most common type of non-scarring hair loss and, affects up to $40 \%$ of women [1]. Classically, FPHL presents as thinning over the top of the head and patients are concerned with a widened part. Unlike male pattern hair loss, the hairline is commonly retained. In the areas of thinning, the terminal, healthy hairs are fewer in number and there are many miniaturized and/or vellus hairs. The pathogenesis of FPHL is complex and likely is triggered by an underlying genetic predisposition and environmental influence. Originally, it was believed that androgens were responsible for FPHL hence the term androgenic alopecia. This notion has been challenged recently as there are reports of women with FPHL that are androgen insensitive. Thus, in this subset of patients, the appearance of FPHL is due to factors other than androgens [2]. On hair shaft biopsy of FPHL, there is often evidence of a microfolliculitis surrounding the hair bulge of the miniaturized hairs and this finding is not found surrounding the terminal hairs, suggesting that inflammation plays a role in FPHL [3].

These findings may explain why some women do not respond to hormonal therapy such as finasteride but do respond to treatments that target inflammation, such as platelet rich plasma (PRP) therapy. PRP therapy involves the use of a patient's own growth factors to influence and support the growth of hair. PRP is made through centrifugation of a patient's blood during an office visit. The least dense layer is the platelets within plasma. Within the platelets are granules that contain more than 20 different growth factors including platelet-derived growth factor, fibroblast growth factor, transforming growth factor, and vascular endothelial growth factor [4]. These growth factors are released into solution by adding calcium chloride or citrate in a process called "activation". Once in solution, the growth factors are concentrated to a level the body never routinely experiences. The growth factor rich solution (PRP) is then injected into areas of thinning at the level of the follicle in the sub-dermis. There are many kits available to physicians to aid in the production of PRP. Some kits link the growth factors to fibrin, known as Platelet Rich Fibrin Matrix or Long-Acting PRP (LA-PRP). In hair loss, I believe this is very advantageous as hair grows slowly and the scalp is vascular. There are studies showing that traditional PRP is washed away in as little as 24 hours where as PRFM may last many weeks if not months slowly releasing growth factors [5]. I believe the duration of effect is the single most important variable in achieving growth when treating hair loss with PRP.

In my practice, I only use LA-PRP and I will pretreat the target area of thinning scalp with a ring block with local anesthesia prior to LA-PRP injection. The LA-PRP session is routinely completed in a single office visit lasting less than 30 minutes, and patients have no downtime from work. The results from a treatment take 2-4 weeks to begin and last for 6 months. Patients will describe first a reduction in shedding, a thickening of the shaft diameter, and possibly new hair growth. After 6 months, the improvement from PRP wanes. Patients are comforted in knowing that all the blood products in this treatment come from their own blood, eliminating the risk of viral infection, allergy, and growth-factor rejection. In my experience in over 500 patients, there has not been an increase in shedding or acute telogen effluvium from treatment at any point in the treatment course. I have also noted that increased frequency of sessions of PRP lead to improved results. There are likely patients who have a genetic predisposition to FPHL and show both a sensitivity to hormonal as well as inflammatory insult. In these patients, combined therapy with minoxidil as well as LAPRP has been very valuable. There are many reports of shedding 
upon starting and stopping minoxidil. This effect seems to be blunted in females when combining treatment with LA-PRP. The current literature is becoming populated with various levels of evidence showing the benefit of PRP for women with FPHL. I would encourage more research into PRFM as an ideal form of LA-PRP for FPHL, as this treatment has been invaluable for my patients.

\section{References}

1. Norwood OT (2001) Incidence of female androgenetic alopecia (female pattern alopecia). Dermatol Surg 27(1): 53-54.

\section{ISSN: 2574-1241}

DOI: 10.26717/BJSTR.2018.11.002036

Benjamin C Paul, MD. Biomed J Sci \& Tech Res

cC (i) This work is licensed under Creative

Submission Link: https://biomedres.us/submit-manuscript.php
2. Orme S, Cullen DR, Messenger AG (1999) Diffuse female hair loss: are androgens necessary? Br J Dermatol 141(3): 521-523.

3. Wilson JD, Griffin JE, Leshin M, Mac Donald PC, JB Stanbury JB, et al. (1983) The androgen resistance syndromes: 5a-reductase deficiency, testicular feminization, and related disorders. McGraw-Hill, New York, USA.

4. Takikawam M, Nakamura S, Nakamura S, Ishirara M, Kishimoto S, et al. (2011) Enhanced Effect of Platelet-Rich Plasma Containing a New Carrier on Hair Growth. Dermatol Surg 37(12): 1721-1729.

5. Sclafani AP (2014) Platelet-rich fibrin matrix (PRFM) for androgenetic alopecia. Facial Plast Surg 30(2): 219-224.

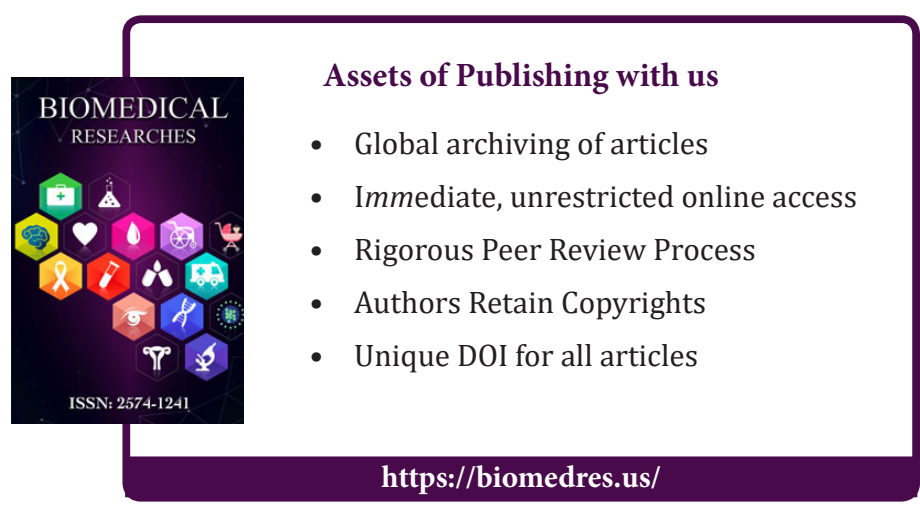

